\title{
ON ALMOST PERIODIC SOLUTIONS OF A CLASS OF DIFFERENTIAL EQUATIONS
}

\author{
G. H. MEISTERS ${ }^{1}$
}

Let $C^{n}$ denote $n$-dimensional complex vector space with vectors $x$ whose components with respect to a given basis are denoted by $x=\left(x^{i}\right)$. Let $\|x\|$ denote the norm of $x$ defined by the equation

$$
\|x\|=\sum_{i=1}^{n}\left|x^{i}\right|
$$

Then $C^{n}$ is a metric space $\left(C^{n}, d\right)$ with metric $d$ defined by the equation

$$
d(x, y)=\|x-y\| .
$$

A set $E$ of real numbers is called relatively dense if there exists a positive real number $l$ such that every interval of length $l$ contains at least one member of the set $E$.

Following Tornehave [4], a continuous function $x(t)$, defined for all real $t$ and having values in a metric space $(X, d)$, is called an almost periodic movement in $X$ if to each positive real number $\epsilon$ there corresponds a relatively dense set of real numbers $\tau$ such that

$$
d(x(t+\tau), x(t)) \leqq \epsilon
$$

for all real $t$.

Similarly, a function $x(k)$, defined on the integers $k=0, \pm 1$, $\pm 2, \cdots$ and having values in a metric space $(X, d)$, will be called an almost periodic sequence in $X$ if to each positive real number $\epsilon$ there corresponds a relatively dense set of integers $\tau$ such that

$$
d(x(k+\tau), x(k)) \leqq \epsilon
$$

for $k=0, \pm 1, \pm 2, \cdots$. In case $(X, d)$ is the space of complex numbers this corresponds to the almost periodic sequences studied by Ingeborg Seynsche [3].

Consider the vector differential equation

$$
d x / d t=F(t, x) \quad-\infty<t<+\infty,
$$

Presented to the Society, April 19, 1958; received by the editors June 4, 1958.

1 The results of this paper constitute part of the dissertation presented by the author for the doctor's degree at Iowa State College. The author wishes to acknowledge his gratitude to Dr. C. E. Langenhop under whose direction the dissertation was prepared. 
where

(a) $F(t, x)$ is an almost periodic movement in $C^{n}$ for each $x$ in some connected open subset $D$ of $C^{n}$, and

(b) $F(t, x)$ satisfies the following Lipschitz condition for all $x$ and $y$ in $D$ :

$$
\|F(t, x)-F(t, y)\| \leqq K\|x-y\| \quad \text { for all real } t .
$$

We shall give a necessary and sufficient condition that a given solution $\phi(t)$ of (1) is almost periodic (=almost periodic movement in $\left.C^{n}\right)$.

Now $F(t, x)$ can be thought of as a family of almost periodic movements in $C^{n}$. For this purpose we shall adopt Tornehave's definition [4, Definition 2, p. 5] of a uniformly continuous family of almost periodic movements. Let $S$ denote a compact metric space and let $(X, d)$ denote an arbitrary metric space with metric $d$. A function $f(t ; v)$ defined for all real $t$ and all $v$ in $S$, and with values in $X$, is called a uniformly continuous family of almost periodic movements in $X$ if:

(a) The function $f(t ; v)$ is an almost periodic movement for each $v$ in $S$, and

(b) To each positive real $\epsilon$ and each $v_{0}$ in $S$ there corresponds a neighborhood $U\left(\epsilon ; v_{0}\right)$ of $v_{0}$ such that $d\left(f(t ; v), f\left(t ; v_{0}\right)\right) \leqq \epsilon$ for all real $t$ and all $v$ in $U\left(\epsilon ; v_{0}\right)$.

Lemma 1. Let $C$ denote a compact subset of $D$, the connected open subset of $C^{n}$ on which $F(t, x)$ is defined. Then $F(t, x)$ with $x$ restricted to $C$ is a uniformly continuous family of almost periodic movements in $C^{n}$.

Proof. Condition (a) of the definition follows immediately from condition (a) of Differential Equation (1). To each positive real number $\epsilon$ and each $x_{0}$ in $C$ choose

$$
U\left(\epsilon ; x_{0}\right)=\left\{x:\left\|x-x_{0}\right\| \leqq \epsilon / K\right\} .
$$

Then by condition (b) of the Differential Equation (1) we have

$$
\left\|F(t, x)-F\left(t, x_{0}\right)\right\| \leqq K\left\|x-x_{0}\right\| \leqq \epsilon
$$

for all $x$ in $U\left(\epsilon ; x_{0}\right)$ and all real $t$. Hence condition (b) of the definition is satisfied.

Lemma 2. If $x(k)$ is an almost periodic sequence in $C^{n}$, then for each positive real number $\epsilon$ the set $T$ of $\epsilon$-translation integers common to $x(k)$ and all the functions of the family $\{F(t, x): x \in C \subset D\}$ is a relatively dense set. 
Proof. Hans Tornehave [4, Theorem 1] has shown that to each uniformly continuous family $f(t ; v)$ of almost periodic movements in a metric space $(X, d)$ there corresponds a real-valued almost periodic function $g(t)$ such that, for each positive $\epsilon$, the set of $\epsilon$-translation numbers common to all the functions of the family $f(t ; v)$ contains the set of $\epsilon$-translation numbers of $g(t)$. Hence to prove the lemma, we have only to show that if $x(k)$ is an almost periodic sequence in $C^{n}$ and $g(t)$ is a real-valued almost periodic function, then for each positive $\epsilon$ the set of $\epsilon$-translation integers common to $x(k)$ and $g(t)$ is a relatively dense set. The proof of this is analogous to the proof that the sum of two almost periodic sequences is again an almost periodic sequence. (Cf. [3]). However, for the reader's convenience, a detailed proof is given here.

Consider S. Bochner's translation function e $(k), k=0, \pm 1, \pm 2, \cdots$, (cf. [1, pp. 8-9]) defined by the equation

$$
e(k)=\sup \{\|x(j+k)-x(j)\|: j=0, \pm 1, \pm 2, \cdots\} .
$$

The following three properties of this function are easy consequences of its definition:

(a) $e(k) \geqq 0, e(0)=0$,

(b) $e(-k)=e(k)$,

(c) $e(j+k) \leqq e(j)+e(k)$.

Furthermore $e(k)$ is an almost periodic sequence. To prove this we write

$$
e(k+\tau) \leqq e(k)+e(\tau)
$$

and

$$
e(k) \leqq e(k+\tau)+e(-\tau)=e(k+\tau)+e(\tau),
$$

and hence

$$
|e(k+\tau)-e(k)| \leqq e(\tau) \quad \text { for all integers } k \text { and } \tau .
$$

For $k=0$ we have

$$
|e(\tau)-e(0)|=e(\tau),
$$

and so

$$
\sup _{k}|e(k+\tau)-e(k)|=e(\tau) .
$$

That is,

$$
\sup _{\boldsymbol{k}}|e(k+\tau)-e(k)|=\sup _{j}\|x(j+\tau)-x(j)\| .
$$


From this last equation we conclude that the set of $\epsilon$-translation integers $\tau$ of $e(k)$ is identical to the set of $\epsilon$-translation integers of $x(k)$. Since the last mentioned set is relatively dense for each positive $\boldsymbol{\epsilon}, e(k)$ is an almost periodic sequence.

Thus to prove the lemma, we need only to show that the set of $\epsilon$-translation integers common to $g(t)$ and $e(k)$ is a relatively dense set. To this end let $\tau_{\theta}$ and $\tau_{\theta}$ denote translation integers of $g(t)$ and $e(k)$, respectively. It is well-known that every almost periodic function possesses a relatively dense set of $\epsilon$-translation integers for each positive real number $\epsilon$. (Cf. [1, Paragraph $4^{\circ}$, p. 54]). Let $l_{0}$ be a positive integer such that every interval $\left[k, k+l_{0}\right], k=0, \pm 1$, $\pm 2, \cdots$, contains an $\epsilon / 2$-translation integer $\tau_{g}$ and an $\epsilon / 2$-translation integer $\tau_{e}$. Let $F_{k}$ denote the interval $\left[k l_{0}, k l_{0}+l_{0}\right]$ for $k=0, \pm 1$, $\pm 2, \cdots$. In each $F_{k}$ select two $\epsilon / 2$-translation integers, $\tau_{b}^{(k)}$ and $\tau_{0}^{(k)}$. The differences $d^{(k)}=\tau_{e}^{(k)}-\tau_{0}^{(k)}$ satisfy

$$
-l_{0} \leqq d^{(k)} \leqq l_{0} \text { for every integer } k \text {. }
$$

Those of the $2 l_{0}+1$ integers $-l_{0}, \cdots, l_{0}$ which occur among the differences $d^{(k)}$ for some $k$ we shall denote by $\left\{i_{1}, i_{2}, \cdots, i_{p}\right\}$, $1 \leqq p \leqq 2 l_{0}+1$. For each $i_{\nu}, \nu=1,2, \cdots, p$, there exists an integer $r_{\nu}$ with smallest absolute value $\left|r_{\nu}\right|$ such that $d^{\left(r_{\nu}\right)}=i_{\nu}$. Let

$$
R=\max \left\{\left|r_{\nu}\right|: \nu=1,2, \cdots, p\right\} .
$$

Choose an integer $L>l_{0}$ so large that the interval $[-L, L]$ contains the interval $F_{R}$. Since $L>l_{0}$, the interval $[m-L, m+L]$ contains at least one of the intervals $F_{k}$ (of length $l_{0}$ ), say $F_{r}$. Select from the intervals $F_{k}$ contained in the interval $[-L, L]$ the one interval $F_{q}$ for which $d^{(q)}=d^{(r)}$. Then

$$
\left(\tau_{a}^{(r)}-\tau_{a}^{(q)}\right)-\left(\tau_{o}^{(r)}-\tau_{o}^{(q)}\right)=d^{(r)}-d^{(q)}=0 .
$$

Hence, since the sum (or difference) of $\epsilon / 2$-translation numbers is an $\epsilon$-translation number, if we set $\tau^{\prime}=\tau_{e}^{(r)}-\tau_{e}^{(a)}=\tau_{0}^{(r)}-\tau_{0}^{(q)}$ then $\tau^{\prime}$ is a common $\epsilon$-translation integer of $e(k)$ and $g(t)$, and since $-L \leqq \tau_{e}^{(e)}$ $\leqq L$ and $m-L \leqq \tau_{e}^{(r)} \leqq m+L$ we have

$$
m-2 L \leqq \tau^{\prime} \leqq m+2 L .
$$

Since $m$ is an arbitrary integer the last inequality proves that the numbers $\tau^{\prime}$ are relatively dense. This completes the proof of Lemma 2.

THEOREM. Let $\phi(t)$ be a vector solution of Differential Equation (1) for all real $t$ and let $D$ contain the closure of the range of $\phi(t)$. Then $a$ necessary and sufficient condition that $\phi(t)$ be an almost periodic movement in $C^{n}$ is that $\phi(k), k=0, \pm 1, \pm 2, \cdots$, be an almost periodic sequence in $C^{n}$. 
Proof. The necessity is trivial since every almost periodic function is an almost periodic sequence when restricted to the integers. This fact is an immediate consequence of $\left[1\right.$, Paragraph $4^{\circ}$, p. 54] and. the fact that the set of $\epsilon$-translation numbers of $\phi(t)$ is identical to the set of $\epsilon$-translation numbers of Bochner's translation function

$$
e(t)=\sup _{s}\|\phi(s+t)-\phi(s)\| . \quad \text { (Cf.[1, pp. 8-9]). }
$$

Thus it is the sufficiency of the condition with which we are primarily concerned here. Consequently for the remainder of the proof we assume that $\phi(k)$ is an almost periodic sequence in $C^{n}$ and that $\phi(t)$ satisfies Differential Equation (1) for all real $t$. With these hypotheses we intend to show that $\phi(t)$ is an almost periodic movement in $C^{n}$.

First of all we shall prove that the range of $\phi(t)$ is a bounded set in $C^{n}$. Clearly

$$
\|\phi(t)\| \leqq\|\phi(t)-\phi(k)\|+\sup _{i}\|\phi(i)\|
$$

where $\sup _{i}\|\phi(i)\|$ is finite since every almost periodic sequence is bounded. Moreover

$$
\begin{aligned}
\|F(t, \phi(k))\| & \leqq\|F(t, \phi(k))-F(t, \phi(0))\|+\|F(t, \phi(0))\| \\
& \leqq K \sup _{\boldsymbol{k}}\|\phi(k)-\phi(0)\|+\sup _{\boldsymbol{t}}\|F(t, \phi(0))\| .
\end{aligned}
$$

Thus $F(t, \phi(k))$ is bounded for all integers $k$ and all real $t$. Let $M$ denote $\sup _{k, t}\|F(t, \phi(k))\|$. Since the Differential Equation (1) can be written as the integral equation

$$
\phi(t)=\phi(0)+\int_{0}^{t} F(s, \phi(s)) d s
$$

we can use (3) and the Lipschitz condition on $F(t, x)$ to obtain the following inequality which holds for all real $t$ in the interval $k \leqq t$ $\leqq k+1$.

$$
\begin{aligned}
\|\phi(t)-\phi(k)\| \leqq & \left\|\int_{k}^{t} F(s, \phi(s)) d s-\int_{k}^{t} F(s, \phi(k)) d s\right\| \\
& +\left\|\int_{k}^{t} F(s, \phi(k)) d s\right\| \\
& \leqq K \int_{k}^{t}\|\phi(s)-\phi(k)\| d s+M .
\end{aligned}
$$


From (4) it follows (cf. [2, Chapter 1, Problem 1]) that

$$
\|\phi(t)-\phi(k)\| \leqq M e^{K} \quad \text { for } k \leqq t \leqq k+1 .
$$

Combining (2) and (5) we now have

$$
\|\phi(t)\| \leqq M e^{K}+\sup _{i}\|\phi(i)\| \quad k \leqq t \leqq k+1
$$

for each integer $k$. Thus $\phi(t)$ is a bounded function.

Now in $\mathrm{C}^{n}$ the closure of a bounded set is bounded and since compact sets in $C^{n}$ are precisely those sets which are both closed and bounded it follows from Lemma 1 that $F(t, x)$, with $x$ restricted to the closure of the range of $\phi(t)$, is a uniformly continuous family of almost periodic movements in $C^{n}$. The range of $\phi(t)$ will be denoted by $R$, and its compact closure by $\bar{R}$. Now by Lemma 2 there exists, for each positive real number $\epsilon$, a relatively dense set $T$ of $\epsilon$-translation integers $\tau$ common to $\phi(k)$ and all the functions of the family $\{F(t, x): x \in \bar{R}\}$. We are now in a position to show that $\phi(t)$ is an almost periodic movement in $C^{n}$.

$\phi(t)$ is certainly continuous for all $t$ since we have assumed it to be differentiable for all $t$. Thus it only remains to be shown that $\phi(t)$ possesses a relatively dense set of $\epsilon$-translation numbers corresponding to each positive $\epsilon$. Consider the following inequality.

$$
\begin{aligned}
\|\phi(t+\tau)-\phi(t)\|= & \left\|\left(\int_{0}^{t+\tau}-\int_{0}^{t}\right) F(s, \phi(s)) d s\right\| \\
= & \left\|\left(\int_{0}^{k+\tau}-\int_{0}^{k}+\int_{k+\tau}^{t+\tau}-\int_{k}^{t}\right) F(s, \phi(s)) d s\right\| \\
\leqq & \|\phi(k+\tau)-\phi(k)\| \\
& +\int_{k}^{t}\|F(s+\tau, \phi(s+\tau))-F(s, \phi(s+\tau))\| d s \\
& +\int_{k}^{t}\|F(s, \phi(s+\tau))-F(s, \phi(s))\| d s .
\end{aligned}
$$

If we now let $\tau$ be an $\epsilon$-translation integer of the relatively dense set $T$ of $\epsilon$-translation integers common to $\phi(k)$ and all $F(t, x)$ for $x$ in $\bar{R}$, the last inequality becomes

$$
\begin{aligned}
\|\phi(t+\tau)-\phi(t)\| & \leqq \epsilon+\epsilon(t-k)+K \int_{k}^{t}\|\phi(s+\tau)-\phi(s)\| d s \\
& \leqq 2 \epsilon+K \int_{k}^{t}\|\phi(s+\tau)-\phi(s)\| d s,
\end{aligned}
$$


for all $t$ in the interval $k \leqq t \leqq k+1$. But then, as before, it follows (cf. [2, Chapter 1, Problem 1]) that

$$
\|\phi(t+\tau)-\phi(t)\| \leqq 2 \epsilon e^{K} \quad k \leqq t \leqq k+1,
$$

for every integer $k$. Thus we see that an $\epsilon$-translation integer common to $\phi(k)$ and all $F(t, x)$ for $x$ in $\bar{R}$ is a $2 \epsilon e^{K}$-translation integer for $\phi(t)$. Therefore $\phi(t)$ is an almost periodic movement in $C^{n}$. This completes the proof of the theorem.

\section{REFERENCES}

1. A. S. Besicovitch, Almost periodic functions, New York, 1954.

2. E. A. Coddington and N. Levinson, Theory of ordinary differential equations, New York, 1955.

3. Ingeborg Seynsche, Zur Theorie der fastperiodischen Zahlfolgen, Rend. Circ. Mat. Palermo vol. 55 (1931) pp. 395-421.

4. Hans Tornehave, On almost periodic movements, Mat.-Fys. Medd. Danske Vid. Selsk. vol. 28, no. 13 (1954) pp. 1-10.

Iowa State College 